\title{
Disposition of the Striated Urethral Sphincter and its Relation to the Prostate in Human Fetuses
}

\author{
Luciano A. Favorito, Luis F. P. Albuquerque, Francisco J. B. Sampaio, Waldemar S. Costa \\ Urogenital Research Unit, State University of Rio de Janeiro, Rio de Janeiro, RJ, Brazil
}

\begin{abstract}
Objective: To describe the arrangement of the muscle fibers of the striated urethral sphincter and its relationship with the prostate during the fetal period in humans.

Materials and Methods: We analyzed 17 prostates from well preserved fresh human fetuses ranging in age from 10 to 31 weeks postconception (WPC). Transversal sections were obtained and stained with Gomori's trichrome and immunolabeled with anti alpha-actin antibody.

Results: We found that the urethral striated sphincter (rabdosphincter) is located on the periphery of the smooth muscle and there was no merge between striated and smooth muscle fibers in any fetal period. In the prostate apex, the striated sphincter shows a circular arrangement and covers completely the urethra externally, whereas adjacent to verumontanum, it looks like a "horseshoe" and covers only the anterior and lateral surfaces of the urethra. Near the bladder neck, in fetuses younger than 20 WPC, we have found striated muscle fibers only at the anterior surface of the prostate, while in fetuses older than $20 \mathrm{WPC}$, the striated muscle covers the anterior and lateral surfaces of the prostate.

Conclusions: The urethral sphincter muscle covers the anterior and lateral surfaces of the urethra in all fetuses older than 20 WPC, close to the bladder neck and at the distal prostate. In the region of the prostate apex, the urethral sphincter covers completely the urethra circularly. The knowledge of the normal anatomy of the urethral sphincter in fetuses could be important to understand its alterations in congenital anomalies involving the base of the bladder, the bladder neck and the proximal urethra.
\end{abstract}

Key words: prostate; urethral sphincter; growth and development; fetuses; anatomy

Int Braz J Urol. 2007; 33: 414-20

\section{INTRODUCTION}

Many anatomic structures are involved in the micturition mechanism such as the bladder base, the prostatic urethra, the membranous urethra and the musculature present in those regions. The disposition of the muscular fibers is important to understand the functional role of those structures that are part of the so-called sphincteric mechanism, acting both in the increase of the urethral pressure and during bladder voiding (1-3).

The main areas involved in the process of urinary continence are located next to the bladder neck and in the distal portion of the prostatic urethra. The latter is formed by three muscular layers; two internal layers (longitudinal and circular) of smooth muscle and 
another external one (circular) formed by striated muscle, being the latter the external striated urethral sphincter itself $(1,4)$.

The anatomic relationship between the external striated urethral sphincter and the prostate apex is decisive in the maintenance of the urinary continence after radical prostatectomy and in many reconstructive surgeries, as for example, surgeries for vesical exstrophy $(5,6)$.

The detailed description of the musculature involved in the urinary continence mechanism is necessary due to the frequency of surgical manipulations on that region, which can harm the sphincter (5). The striated sphincter was well described recently through techniques of computerized reconstruction and magnetic resonance (7-9). The structure of the striated sphincter, disposed like a collar, and its alterations during embryo and fetal development could explain the higher incidence of vesicoureteral reflux in boys when compared to girls $(10,11)$.

The studies that assess the disposition of the external striated urethral sphincter or the distribution of the smooth and striated muscle in the initial phases of the human development are scarce $(11,12)$. The objectives of this work are to describe the localization and direction of the striated fibers of the external striated urethral sphincter during the human fetal period and to analyze the relationships between the smooth and striated muscle layers with the prostatic urethra.

\section{MATERIALS AND METHODS}

We studied 17 blocks containing the prostate, the urethra and the periprostatic musculature, obtained from well preserved fresh human fetuses, ranging in age from 10 to 31 weeks postconception (WPC). The fetuses died of causes unrelated to the urogenital tract and no external evidence of congenital malformations was detected. The gestational age of the fetuses was estimated according to the foot length principle (1316), which is nowadays this is the most acceptable method to estimate the fetal age. The relationships between the fetal age, the weight and the vertex-coccyx length (VC) is shown on Table-1.
The fetal pelvis was carefully dissected with the aid of a X2.5 magnifying glass. After dissection, the prostate was removed and immersed in a Bouin solution for 48 to 72 hours. Afterwards the material was immersed in paraffin and sections of $5 \mu \mathrm{m}$ thick were made. The sections were stained with Gomori's trichrome to verify the integrity of the specimens and to demonstrate the striated musculature.

The immunostainning procedures were performed with Zymed ${ }^{\circledR}$ primary monoclonal anti-alpha smooth muscle actin with appropriate positive and negative controls. Briefly, sections from formalin fixed, paraffin embedded samples were de-waxed, hydrated in a graded series of ethanol solutions of decreasing concentrations until the solution was all water and then washed in phosphate buffered saline (PBS) for $5 \mathrm{~min}$ utes. The sections were treated for 10 minutes with $3 \%$ hydrogen peroxide solution in methanol to block endogenous peroxidase activity. The sections were washed in three drops PBS, incubated in a humid chamber for 10 minutes with $1 \%$ goat serum, and then incubated a humid chamber with primary antibody predilute 30 a 60 minuts.

Table 1 - The table presents fetuses' ages in weeks post conception, the weights in grams and the vertex-coccyx length $(V C)$ in millimeters.

\begin{tabular}{lrrr}
\hline Fetus & Age & Weight & VC Length $(\mathrm{mm})$ \\
\hline 1 & 10 & 88 & 64 \\
2 & 14 & 140 & 125 \\
3 & 14 & 103 & 130 \\
4 & 16 & 230 & 160 \\
5 & 18 & 311 & 175 \\
6 & 18 & 340 & 180 \\
7 & 19 & 390 & 185 \\
8 & 20 & 350 & 180 \\
9 & 20 & 71 & 190 \\
10 & 20 & 381 & 180 \\
11 & 21 & 531 & 220 \\
12 & 25 & 698 & 230 \\
13 & 25 & 805 & 240 \\
14 & 25 & 751 & 240 \\
15 & 29 & 1190 & 270 \\
16 & 30 & 1800 & 290 \\
17 & 31 & 1790 & 280 \\
\hline
\end{tabular}


Subsequently, the sections washed in three drops PBS and incubated at room temperature in a humid chamber with the biotinylated secondary antibody (Histostain-plus Kits Zymed) for 20 minutes, washed in three drops PBS and incubated at room temperature in a chamber with streptavidin- peroxidase-conjugate for 10 minutes. The sections were washed in three drops PBS and revealed by treating with a 3'3-diaminobenzidine tetrahydrochloride solution containing $0.1 \%$ volume in volume hydrogen peroxide, and washed in distilled water, dehydrated in an increasing concentration series of ethanol solutions and mounted with rapid mounting media for microscopy.

The present study was approved by the Research Ethics Committee of our institution.

\section{RESULTS}

The striated musculature is the most external layer of the prostate, being clear the separation of this layer from the extraprostatic adjacent tissue (Figure1). Internally, the striated muscle is in contact with the prostate stroma, mainly formed by collagen and smooth muscle. Also here the transition is evident even though not in a linear way as it is seen in relation to the extraprostatic tissue but through extensions both of conective tissue rich in collagen and the smooth

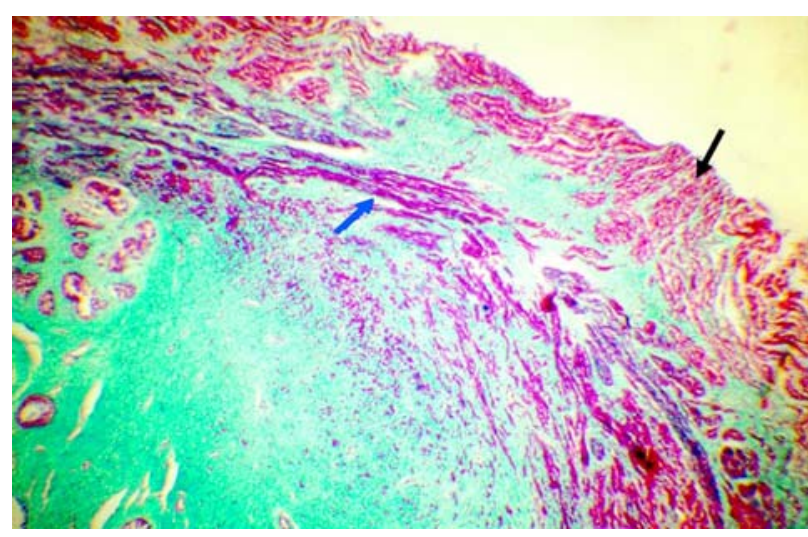

Figure 1-Fetus with 20 WPC. Section through the prostate base. Striated muscle is located externally (black arrow) and smooth muscle is located internally (blue arrow). Gomori's trichrome, X100. muscle, in direction of the striated muscle. Various regions present these extensions, however, it was never observed a mixture between striated and smooth muscle fibers.

In all fetal ages studied we have detected muscle fibers extending from the prostate apex to the base. These fibers did not totally involve the prostate and were more evident anteriorly, presenting a horseshoe disposition. The muscle fibers were disposed in an external layer formed by striated muscle and an internal layer formed by smooth muscle fibers (Figure-2).

We have found striated fibers in the prostate lateral surfaces only in fetuses with more than 20 WPC. We did not find any striated muscle in the mid and proximal posterior surface of the prostate in none of the cases. At the prostate apex the striated muscle surrounds all the urethra and its fibers are circularly disposed (Figure-3).

In a fetus with 10 WPC we have observed an important concentration of muscular fibers restricted to the ventral region of the prostate (Figure$4)$. As the fetuses growth we observed an extension of the muscle fibers for the lateral surfaces of the prostate with a progressive increase from 18 to 25 WPC (Figure-5). The prostates of the fetuses with more advanced age presented more smooth muscle in their lateral surfaces (Figure-6).

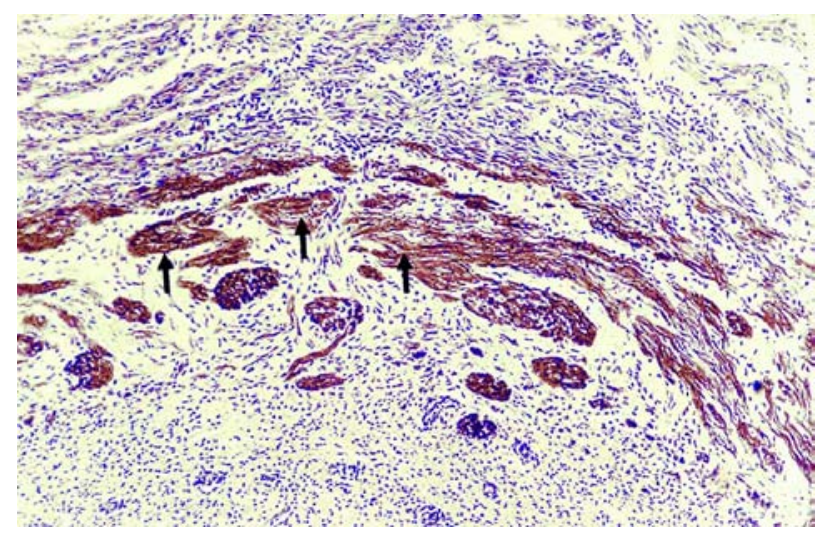

Figure 2 - Fetus with 20 WPC. Section through the prostate base. The smooth muscle fibers can be observed restricted to the anterior and lateral surfaces (arrows). Immunolabelling with anti alpha-actin, X100. 


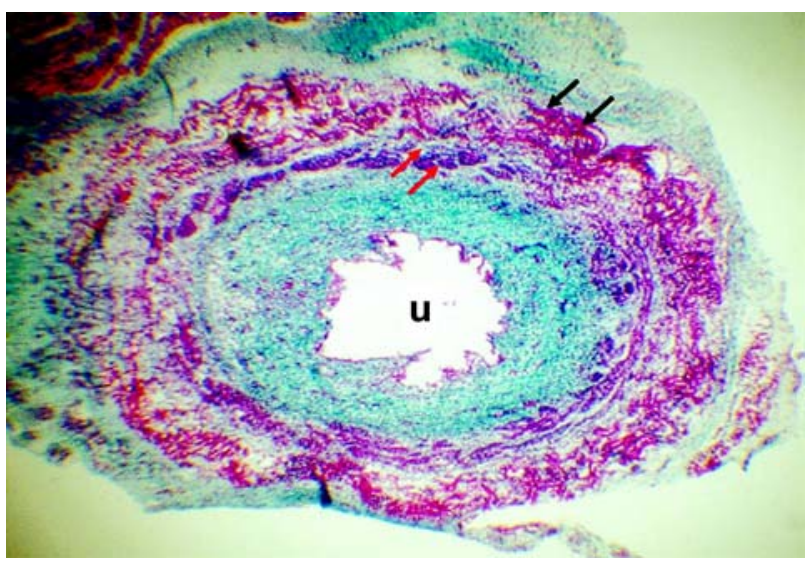

Figure 3-Fetus with 10 WPC. Section at the level of the prostate apex where we can observe that striated muscle layer (black arrows) as well as smooth muscle layer (red arrows) involve the urethra (u) circumferentially. Gomori's trichrome, X40.

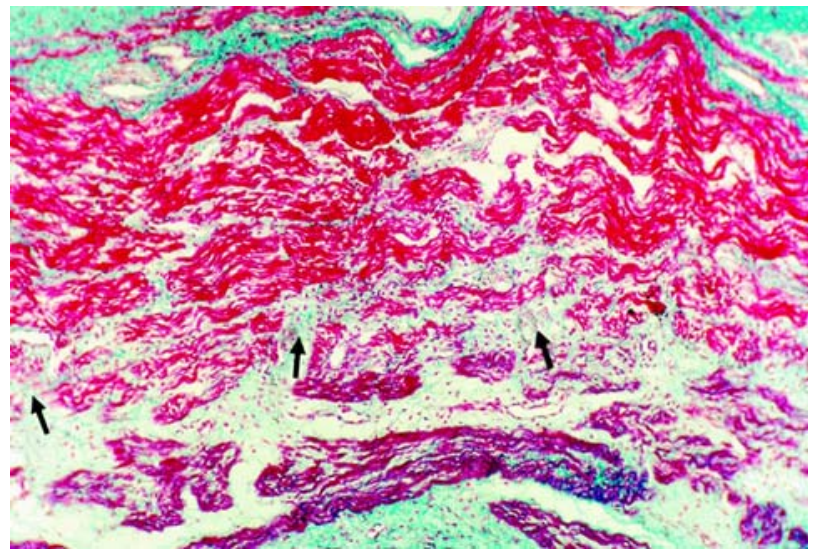

Figure 5-Fetus with 25 WPC. Section at the level of the prostate base where the lateral region of the gland can be observed. The transition line between the layers of striated muscle and smooth muscle, formed by digitations of collagen (arrows) can be clearly evidenced. Gomori's trichrome, X100.

In the specimens where an imunohistochemistry with anti-alpha actin antibody was performed, that evidence the smooth musculature, we confirm that the muscular fibers that were not labelled (striated muscle) were localized more externally. The stained labelled fibers (smooth muscle), on the other hand were localized more internally and in close contact with the prostatic capsule (Figure-2).

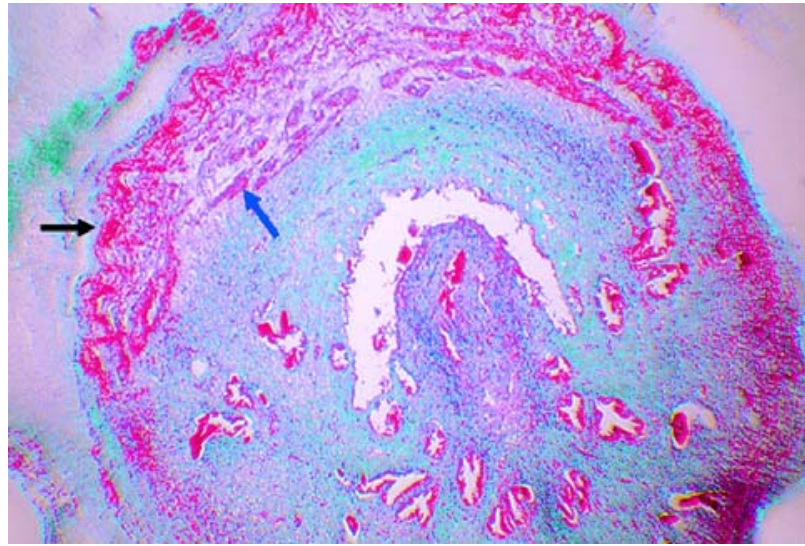

Figure 4 - Fetus with 10 WPC. Section at the level of the veromontanum depicts the external striated urethral sphincter (black arrow) and the smooth muscle fibers (blue arrow). Notice that the muscle does not involve all the circumference of the prostate, being restricted mainly to the anterior region. Gomori's trichrome, X40.

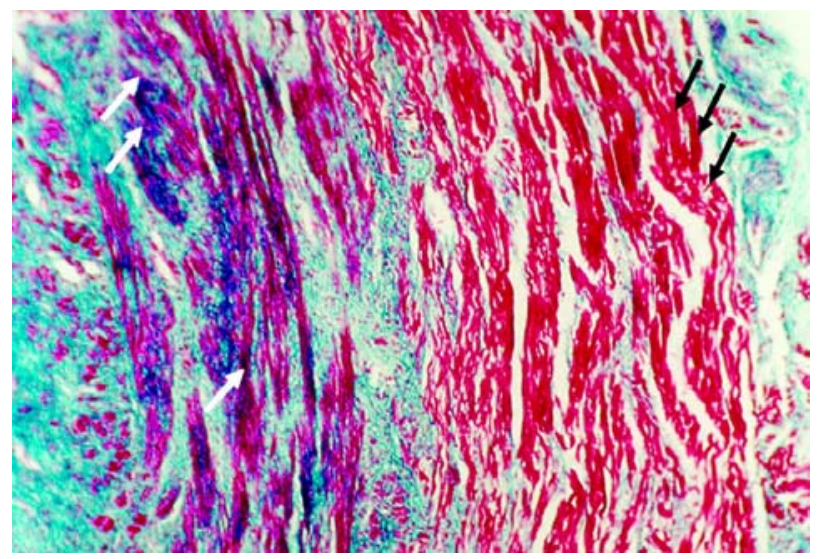

Figure 6-Fetus with 30 WPC. Section at the level of the veromontanum, where in this older fetus we can now observe the extension of the striated muscle (black arrows) and the smooth one (white arrows) for the lateral region of the prostate. Gomori's trichrome, X100.

\section{COMMENTS}

The components of the bladder neck, distal third of the prostatic urethra, and of the prostate apex actively participate in the process of urinary continence. The circular smooth muscle of the bladder neck is a direct extension of the longitudinal layer of the detrusor muscle. 
During the bladder distention there is a strecth of the longitudinal muscular fibers and this increase in tension is transmitted to bladder neck circular fibers, determining the closure of the urethra. On the other hand, with the descent of the bladder neck during micturition, those circular fibers assume an oblique direction (2). The circular layer of smooth muscle is prominent in the regions of the prostate base and middle prostate, while the longitudinal layer is more evident in the distal portion (18). In our study we have observed a circular smooth muscle layer localized mainly at the prostate base, while the longitudinal muscle layer was more evident at the prostate apex.

Previous studies have evidenced that the circular striated fibers of the distal sphincter assume a longitudinal direction when reaching the lateral surface of the prostate, ascending to the bladder neck $(4,12)$. Other works show the presence of striated muscle near the bladder neck, however in the lateral and anterior positions $(2,4,12)$. In our study we observe near the bladder neck, striated fibers localized in the prostate anterior and lateral regions. In none of the sections performed at the base of the protate we observed striated muscle on its posterior surface.

The striated musculature of the urethral sphincter surrounds the urethra in the region of the prostate apex, but does not surround the prostate in the veromontanum level as well as in the prostate base, presenting a horseshoe aspect, confirming the findings of previous studies performed in fetuses $(11,19)$. Our findings agree with those of Yucel \& Baskin (11), that evidenced a change in the development pattern of striated sphincter during the fetal period and the horseshoe aspect of the musculature in the superior portions of the prostate. Ludwikowski et al. (10) reported that they did not find changes in the development pattern of the striated sphincter during the fetal period studied and did not find at any age the sphincter surrounding the prostatic apex, that are against our findings.

During the performance of the endoscopic surgeries of the prostate a resection of the supramontanal portion of the prostatic urethra is performed, that is the region where generally occurs the growth of the adenoma (20). The quantity of striated muscle in this region is inferior to that observed in the inferior portions of the prostate. The region above the veromontanum is mainly constituted by smooth muscle, however this musculature is little damaged, since the smooth muscle is compressed in the direction of the surgical capsule by the adenoma. In this way, the incidence of incontinence after the transurethral resection is minimal.

The longitudinal and circular portions of the striated sphincter form an arch when analyzed together. The urethra penetrates in the anterior region of the bladder base and descends obliquely through the prostate crossing this arch of striated muscle. The striated muscle circular portion is separated from the urethra by mucosal glands and smooth muscle. The sphincter fibers are transversal in comparison with the longitudinal fibers of pubovesical ligaments that are placed anterior to the sphincter, separated from those only by a narrow band of conjunctive tissue of the retropubic space $(5,12,21)$.

In adults, the anterior and posterior surfaces of the sphincter are close related to an extensive prostatic vascular plexus. The integrity of the muscle as a distinctive structure is lost due to the advancement of the vascular plexus, making it difficult to describe this structure (12). In fetuses, we found a clear separation between the striated muscle and the prostate peripheral tissue, similar to a capsule.

Oerlich (12) describes that the muscle fibers of the mesenchyme start to present striations in fetuses with $115 \mathrm{~mm} \mathrm{VC} \mathrm{length,} \mathrm{and} \mathrm{a} \mathrm{complete} \mathrm{distinc-}$ tion of striated and smooth fibers is observed in fetuses with $245 \mathrm{~mm} \mathrm{VC}$ length. We have found striations in all prostates studied from the age of $10 \mathrm{WPC}$ ( $64 \mathrm{~mm} \mathrm{VC}$ length), and those fibers are different from the smooth muscle fibers in a very evident way. The presence of sphincteric muscle in the prostatic region in fetuses of the third trimester was also noticed by Ludwidowski et al. (10) and by Sebe et al. (19).

Striated muscle fibers are continuous and inseparable from the smooth fibers of the urethra and interdigitations occur in the contact plane between the two types of fibers. There is no kind of fascia between the two areas $(12,22-24)$. In our sample we have found the same interdigitations between the two muscular types, even though no fascial structure has been found. 
At the veromontanum level and in the prostate base we have found an overlay in the lateral surfaces by the striated urethral sphincter. In the prostate apex, the fibers are disposed with a circular orientatin, while in the middle prostate did not present a defined direction. The relationship between the prostate and the external urethral striated sphincter changes with the prostate development, mainly concerning their lateral surfaces. With the lateral growth of the lobes, that depends upon individual characteristics, the striated fibers localized over the lateral surfaces become more separate. The extension in which the lateral surfaces will be overlayed by such fibers is variable and will depend on the development of the lateral lobes(12).

We conclude that the external striated sphincter surrounds all the urethra at the prostate apex and involves the anterior and lateral surfaces. Near the bladder neck the musculature is found only at the anterior face of the prostate in fetuses until 20 WPC, while in fetuses with more than 20 WPC we can observe an extension of the striated fibers to the lateral surfaces. The direction of the striated fibers is predominantly transversal at the prostate apex, at the anterior surface of the prostate base and at the middle of the prostate. However, at the prostate lateral region of the middle prostate its disposition is aleatory.

\section{ACKNOWLEDGEMENTS}

This research was supported by the National Council of Scientific and Technological Development (CNPq) and by the Rio de Janeiro Foundation for Research Support (FAPERJ), Brazil. Waldemar S. Costa, Luciano A. Favorito and Francisco J. B. Sampaio contributed equally to the research and manuscript preparation.

\section{CONFLICT OF INTEREST}

None declared.

\section{REFERENCES}

1. Presti JC Jr, Schmidt RA, Narayan PA, Carroll PR, Tanagho EA: Pathophysiology of urinary incontinence after radical prostatectomy. J Urol. 1990; 143: 975-8.

2. Light JK, Rapoll E, Wheeler TM: The striated urethral sphincter: muscle fibre types and distribution in the prostatic capsule. Br J Urol. 1997; 79: 539-42.

3. Whitmore I, Gosling JA, Gilpin SA: A comparison between the physiological and histochemical characterisation of urethral striated muscle in the guinea pig. Pflugers Arch. 1984; 400: 40-3.

4. Manley CB Jr: The striated muscle of the prostate. J Urol. 1966; 95: 234-40.

5. Myers RP, Goellner JR, Cahill DR: Prostate shape, external striated urethral sphincter and radical prostatectomy: the apical dissection. J Urol. 1987; 138: 543-50.

6. Hauri D, Heinzelmann M, Konstantinidis K: Radical prostatectomy in cases of prostatic carcinoma: the problem of postoperative urinary incontinence. Urol Int. 1988; 43: 257-64.

7. Myers RP, Cahill DR, Kay PA, Camp JJ, Devine RM, King BF, et al.: Puboperineales: muscular boundaries of the male urogenital hiatus in 3D from magnetic resonance imaging. J Urol. 2000; 164: 1412-5.

8. Strasser H, Bartsch G: Anatomy and innervation of the rhabdosphincter of the male urethra. Semin Urol Oncol. 2000; 18: 2-8.

9. Brooks JD, Chao WM, Kerr J: Male pelvic anatomy reconstructed from the visible human data set. J Urol. 1998; 159: 868-72.

10. Ludwikowski B, Oesch Hayward I, Brenner E, Fritsch $\mathrm{H}$ : The development of the external urethral sphincter in humans. BJU Int. 2001; 87: 565-8.

11. Yucel S, Baskin LS: An anatomical description of the male and female urethral sphincter complex. J Urol. 2004; 171: 1890-7.

12. Oelrich TM: The urethral sphincter muscle in the male. Am J Anat. 1980; 158: 229-46.

13. Hern WM: Correlation of fetal age and measurements between 10 and 26 weeks of gestation. Obstet Gynecol. 1984; 63:26-32.

14. Mercer BM, Skalar S, Shariatmadar A, Gillieson MS, D'alton ME: Fetal foot length as a predictor of gestational age. Amer J Obst Gynec, 1987; 156: 350-356.

15. Platt LD, Medearis AL, DeVore GR, Horenstein JM, Carlson DE, Brar HS: Fetal foot length: relationship to menstrual age and fetal measurements in the second trimester. Obstet Gynecol. 1988; 71: 526-31. 


\section{Urethral Sphincter and Human Fetus Prostate}

16. Sampaio FJ, Favorito LA: Analysis of testicular migration during the fetal period in humans (10 to 35 weeks postconception). J. Urol. 1988; 159: 540-2.

17. Hsu SM, Raine L, Fanger H: Use of avidin-biotin-peroxidase complex (ABC) in immunoperoxidase techniques: a comparison between $\mathrm{ABC}$ and unlabeled antibody (PAP) procedures. J Histochem Cytochem. 1981; 29: 577-80.

18. Sant GR: The anatomy of the external striated urethral sphincter. Paraplegia. 1972; 10: 153-6.

19. Sebe B, Schwentner C, Oswald J, Radmayr C, Bartsch G, Fritsch H: Fetal development of striated and smooth muscle sphincters of the male urethra from a common primordium and modifications due to the development of the prostate: an anatomic and histologic study. Prostate. 2005 ; 62: 388-93.
20. Hutch JA, Rambo OS Jr: A study of the anatomy of the prostate, prostatic urethra and the urinary sphincter system. J Urol. 1970; 104: 443-52.

21. Haines RW: The striped compressor of the prostatic urethra. Br J Urol. 1969; 41: 481-93.

22. Kokoua A, Homsy Y, Lavigne JF, Williot P, Corcos J, Laberge I, et al.: Maturation of the external urinary sphincter: a comparative histotopographic study in humans. J Urol. 1993; 150: 617-22.

23. Elbadawi A, Mathews R, Light JK, Wheeler TM: Immunohistochemical and ultrastructural study of rhabdosphincter component of the prostatic capsule. J Urol. 1997; 158: 1819-28.

24. Dorschner W, Biesold M, Schmidt F, Stolzenburg JU: The dispute about the external sphincter and the urogenital diaphragm. J Urol. 1999; 162: 1942-5.

\author{
Correspondence address: \\ Dr. Luciano A. Favorito \\ State University of Rio de Janeiro, UERJ \\ Urogenital Research Unit \\ Av. 28 de Setembro, No. 87, fundos \\ Rio de Janeiro, RJ, 20551-030, Brazil \\ Fax: + 5521 2587-6121 \\ E-mail: favorito@urogenitalresearch.org
}

Accepted after revision: February 26, 2007 